Supporting Information

\title{
Synthesis and Spectroscopy of Emissive, Surface-Modified, Copper-Doped Indium Phosphide Nanocrystals
}

\author{
M. Elizabeth Mundy, Forrest W. Eagle, Kira E. Hughes ${ }^{\dagger}$, Daniel R. Gamelin, and Brandi M. Cossairt* \\ University of Washington, Department of Chemistry, Box 351700, Seattle, WA 98195-1700.
}

\section{Experimental Methods}

All glassware was dried in a $160{ }^{\circ} \mathrm{C}$ oven overnight prior to use. All reactions were run under an inert atmosphere of nitrogen using a glovebox or standard Schlenk techniques. Zinc chloride (>98\%), trisdiethylaminophosphine (97\%), copper (II) chloride ( $\geq 98 \%$ ), indium (III) chloride (97\%), indium (III) bromide (99\%), and anhydrous isopropanol were purchased from Millipore-Sigma, stored in a nitrogen glovebox or desiccator, and used without further purification. Indium iodide (99\%), copper (I) chloride (anhydrous 97\%+), and copper (II) bromide (99\%) were purchased from Strem Chemicals Inc., stored in a nitrogen glovebox or desiccator, and used without further purification. All other solvents including oleylamine, pentane, and toluene were purchased from Sigma Aldrich Chemical Co., dried over $\mathrm{CaH}_{2}$, distilled, and stored over $4 \AA$ sieves in a nitrogen glovebox. Omni Trace nitric acid was purchased from EMD Millipore and used without purification. 18.2 M $\Omega$ water was collected from an EMD Millipore water purification system. ${ }^{31} \mathrm{P}$ NMR spectra were collected on a $500 \mathrm{MHz}$ Bruker Avance spectrometer. UV-Vis spectra were collected on a Cary 5000 spectrophotometer from Agilent. TEM images were collected on a FEI Tecnai G2 F20 microscope using an ultrathin carbon film on holey carbon purchased from Ted Pella Inc. P-XRD diffractograms were collected a Bruker Microfocus instrument.

Luminescence spectra were collected on an Edinburgh FLS 1000 fluorimeter. Photoluminescence quantum yields were measured with a Hamamatsu integrating sphere.

\section{Standard doped indium phosphide reaction procedure}

$0.45 \mathrm{mmol}$ of an indium halide, $2.2 \mathrm{mmol} \mathrm{ZnCl}_{2}$, and $5 \mathrm{~mL}$ of dried and distilled oleylamine were added to a $25 \mathrm{~mL}$ 3-neck flask. The solution was then placed under vacuum at $120^{\circ} \mathrm{C}$ and degassed for 1 hour. The vessel was then placed under an inert atmosphere and heated to $180{ }^{\circ} \mathrm{C}$. Once the reaction temperature was reached, $0.45 \mathrm{~mL}$ of tris-diethylaminophosphine $(1.6 \mathrm{mmol})$ was rapidly injected. The reaction was allowed to proceed for 5 minutes after which the previously prepared copper halide solution was slowly injected $(2 \mathrm{~mL}$ at $4 \mathrm{~mL} / \mathrm{h})$. The flask was then cooled down to room temperature before being moved into a nitrogen glovebox for purification. The nanocrystals were precipitated with anhydrous methanol or isopropanol, centrifuged at $7830 \mathrm{rpm}$, and suspended in toluene. This procedure was repeated 5 times before any additional sample analysis was performed.

\section{Thin shelled doped indium phosphide reaction procedure}

$0.23 \mathrm{mmol}$ of an indium halide, $1.1 \mathrm{mmol}$ of $\mathrm{ZnCl}_{2}$, and $2.5 \mathrm{~mL}$ of dried and distilled oleylamine were added to a $15 \mathrm{~mL} 3$-neck flask. The solution was then placed under vacuum at $120{ }^{\circ} \mathrm{C}$ and degassed for 1 hour. The vessel was then placed under an inert atmosphere and heated to $180{ }^{\circ} \mathrm{C}$. Once the reaction temperature was reached, $0.23 \mathrm{~mL}$ of tris-diethylaminophosphine $(0.8 \mathrm{mmol})$ was rapidly injected. The reaction was allowed to proceed for 20 minutes after which $0.5 \mathrm{~mL}$ of the previously prepared $1 \mathrm{M}$ $\mathrm{TOP}=\mathrm{Se}$ was slowly injected. At 60 minutes the temperature was increased to $200{ }^{\circ} \mathrm{C}$ and held there for 
an hour. At 120 minutes, the temperature was decreased to $150{ }^{\circ} \mathrm{C}$. Once temperature was reached the copper halide solution was slowly injected $(1 \mathrm{~mL}$ at $2 \mathrm{~mL} / \mathrm{h})$. After the injection had completed the temperature was increased to $210{ }^{\circ} \mathrm{C}$ and held there for 1.5 hours. The flask was then cooled down to room temperature before being moved into a nitrogen glovebox for purification. The nanocrystals were precipitated with anhydrous methanol or isopropanol, centrifuged at $7830 \mathrm{rpm}$, and suspended in toluene. This procedure was repeated 5 times before any additional sample analysis was performed.

Modified shelling procedures to grow a thicker ZnSeS shell used a method adapted Economic and SizeTunable Synthesis of InP/ZnE (E = S, Se) Colloidal Quantum Dots. Chem. Mater., 2015, 27, 4893-4898 where $\mathrm{TOP}=\mathrm{Se}$ was used in place of $\mathrm{TOP}=\mathrm{S}$ in the first chalcogenide injection.

\section{Zinc stearate treatment procedure}

$0.1 \mathrm{mmol}$ zinc stearate was degassed in a $15 \mathrm{~mL}$ flask. Half of a previously prepared $\mathrm{Cu}$ :InP nanocrystal reaction at the $0.45 \mathrm{mmol}$ scale was precipitated once and then resuspended in $4 \mathrm{~mL}$ of 1 -octadecene. The solution containing approximately $0.2 \mathrm{mmol}$ of indium was injected into the flask containing the zinc stearate at room temperature. The entire solution was gently heated while stirring to $100{ }^{\circ} \mathrm{C}$ and held at that temperature for 2 hours. The flask was then cooled down to room temperature before being moved into a nitrogen glovebox for purification. The nanocrystals were precipitated with anhydrous methanol or isopropanol, centrifuged at $7830 \mathrm{rpm}$, and suspended in toluene. This procedure was repeated 5 times before any additional sample analysis was performed.

\section{Sample preparation for characterization}

For PXRD samples, insoluble powders were placed onto a piece of double-sided tape on a silicon $\langle 100\rangle$ single crystal wafer. Solvent was removed from colloidal samples until dryness was achieved and then the

paste was spread on a silicon $\langle 100\rangle$ single crystal wafer. For TEM, samples were suspended in toluene and then drop cast onto a TEM grid and placed under vacuum overnight prior to imaging to ensure full solvent evaporation. ICP-OES: An aliquot of QD solution was dried to generate solid powder. The supernatant was discarded and hydrogen peroxide, followed by an equivalent amount of trace metal grade nitric acid (67\%) was added to the powder and digested overnight. 18.2 $\mathrm{M} \Omega$ water was added to the sample to dilute to a total of $2 \%$ acid concentration.

\section{Photophysical characterization methods}

Room-temperature TRPL measurements were performed by exciting colloidal NCs at $365 \mathrm{~nm}$ via a Coherent Inc./Light Source OPerA optical parametric amplifier, power measured at $\sim 50 \mu \mathrm{W}$. Timeresolved PL spectra were collected using a Hamamatsu streak camera with a synchroscan unit. Transient Absorption measurements were performed using an EOS unit from Ultrafast Systems at the University of Washington's Molecular Analysis Facility. The pump wavelength of $365 \mathrm{~nm}$ was generated via a Coherent Inc./Light Source OPerA optical parametric amplifier, with a power of $\sim 50 \mu \mathrm{W}$ measured through a $200 \mu \mathrm{m}$ pinhole. The probe white light was generated using an external Q-switched Nd:YAG laser with an electronic delay. The collinear pump and probe beams overlapped at the sample. 

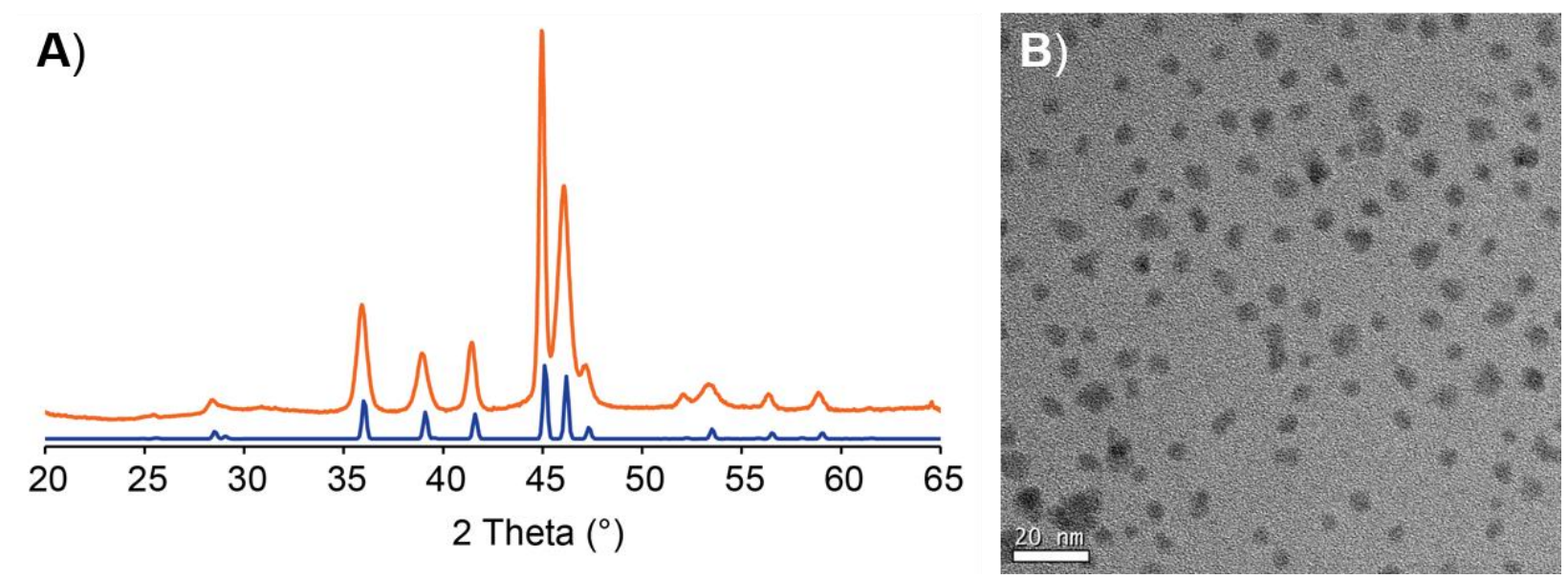

Figure S1. (A) P-XRD spectrum of product of reaction of $\mathrm{P}\left(\mathrm{NEt}_{2}\right)_{3}$ and $\mathrm{CuCl}_{2}$ (orange) with $\mathrm{Cu}_{3} \mathrm{P}$ standard pattern (blue, pdf 01-071-2261 ICSD) and (B) TEM image showing clear evidence of crystalline copper phosphide.

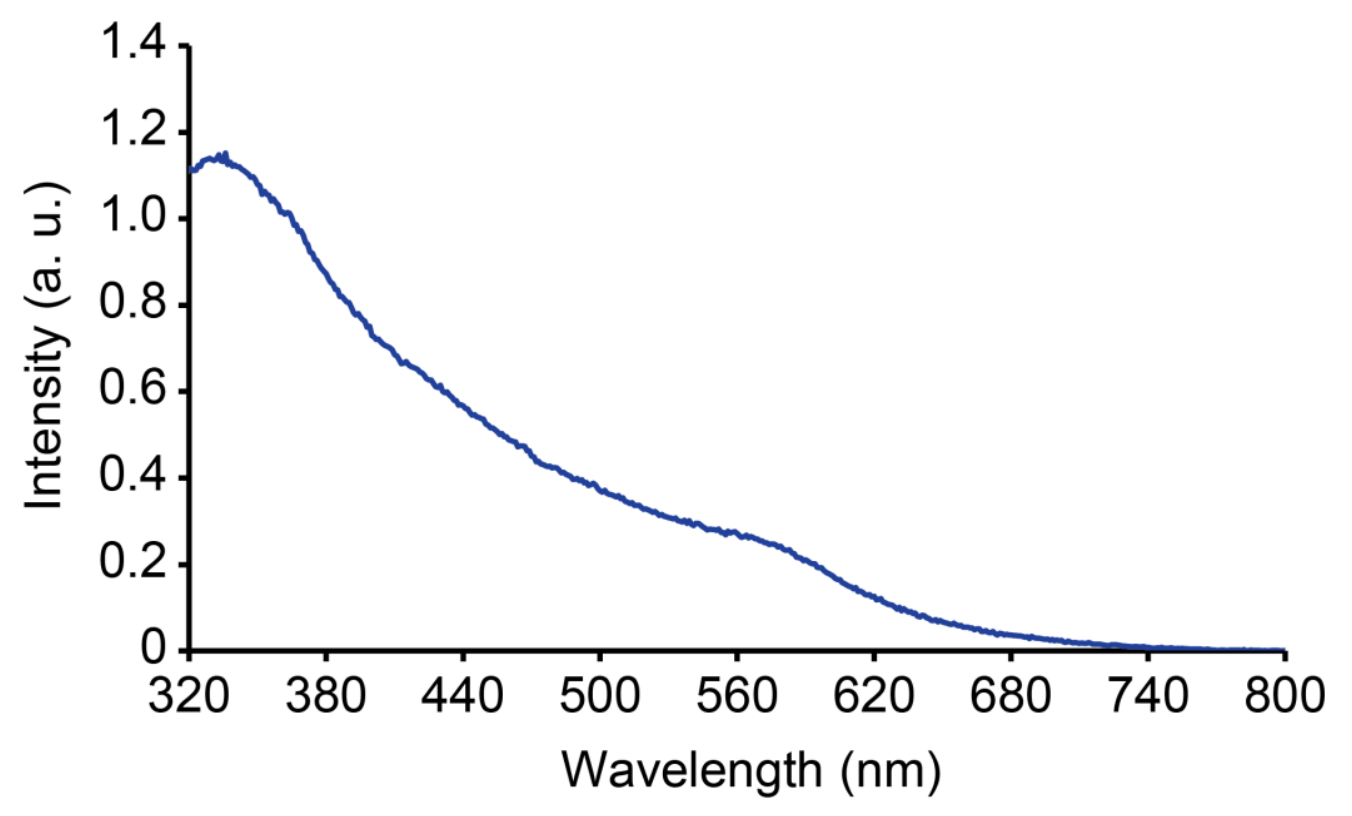

Figure S2. Photoluminescence excitation monitored at $825 \mathrm{~nm}$ taken on a purified sample of $\mathrm{Cu}^{+}: \mathrm{InP}$ $\mathrm{NCs}$ prepared from $\mathrm{InCl}_{3}$. The feature at $585 \mathrm{~nm}$ corresponds to the excitonic absorbance in the UV-Vis spectrum shown in Figure 1B. 


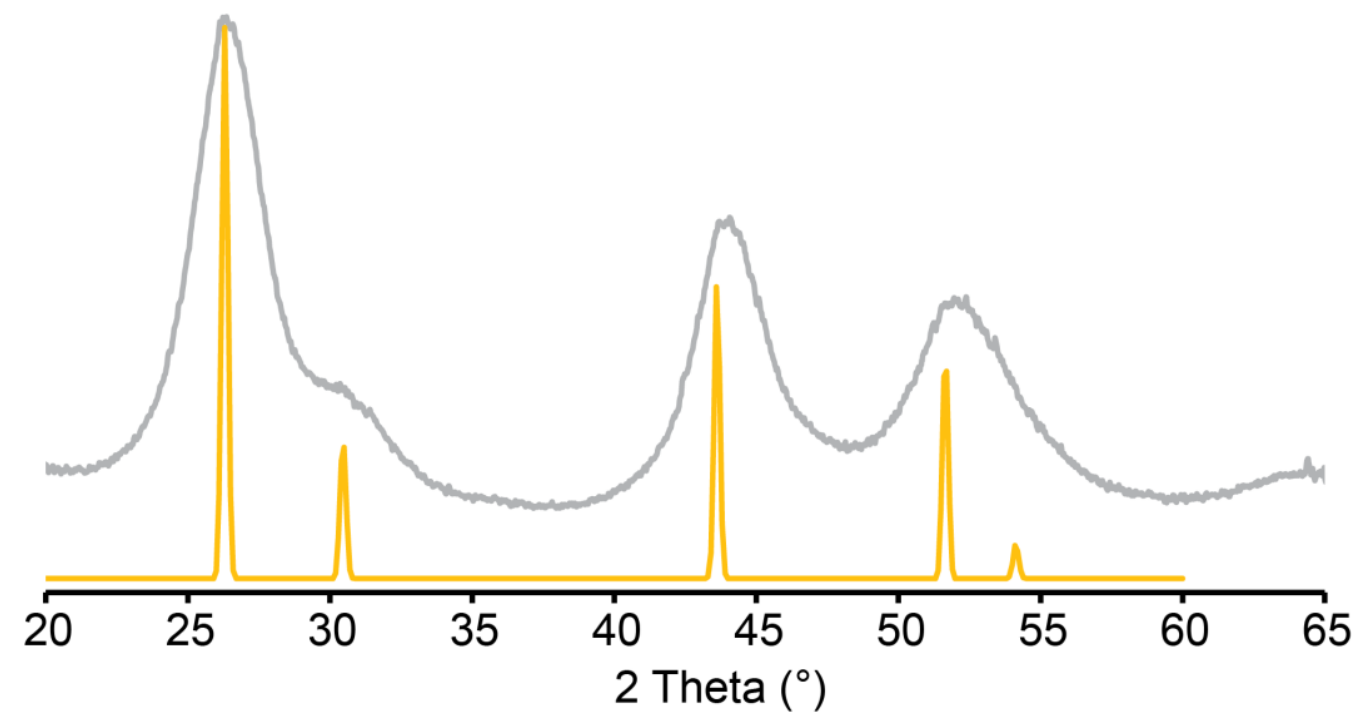

Figure S3. P-XRD spectrum (gray) of nanocrystals formed by reacting $\mathrm{InCl}_{3}$ and $\mathrm{P}\left(\mathrm{NEt}_{2}\right)_{3}$ according to Scheme 1 and doped using $\mathrm{CuBr}_{2}$ instead of $\mathrm{CuCl}_{2}$ as the copper precursor. Reference bulk InP pattern (yellow, pdf 01-070-2513 ICSD).
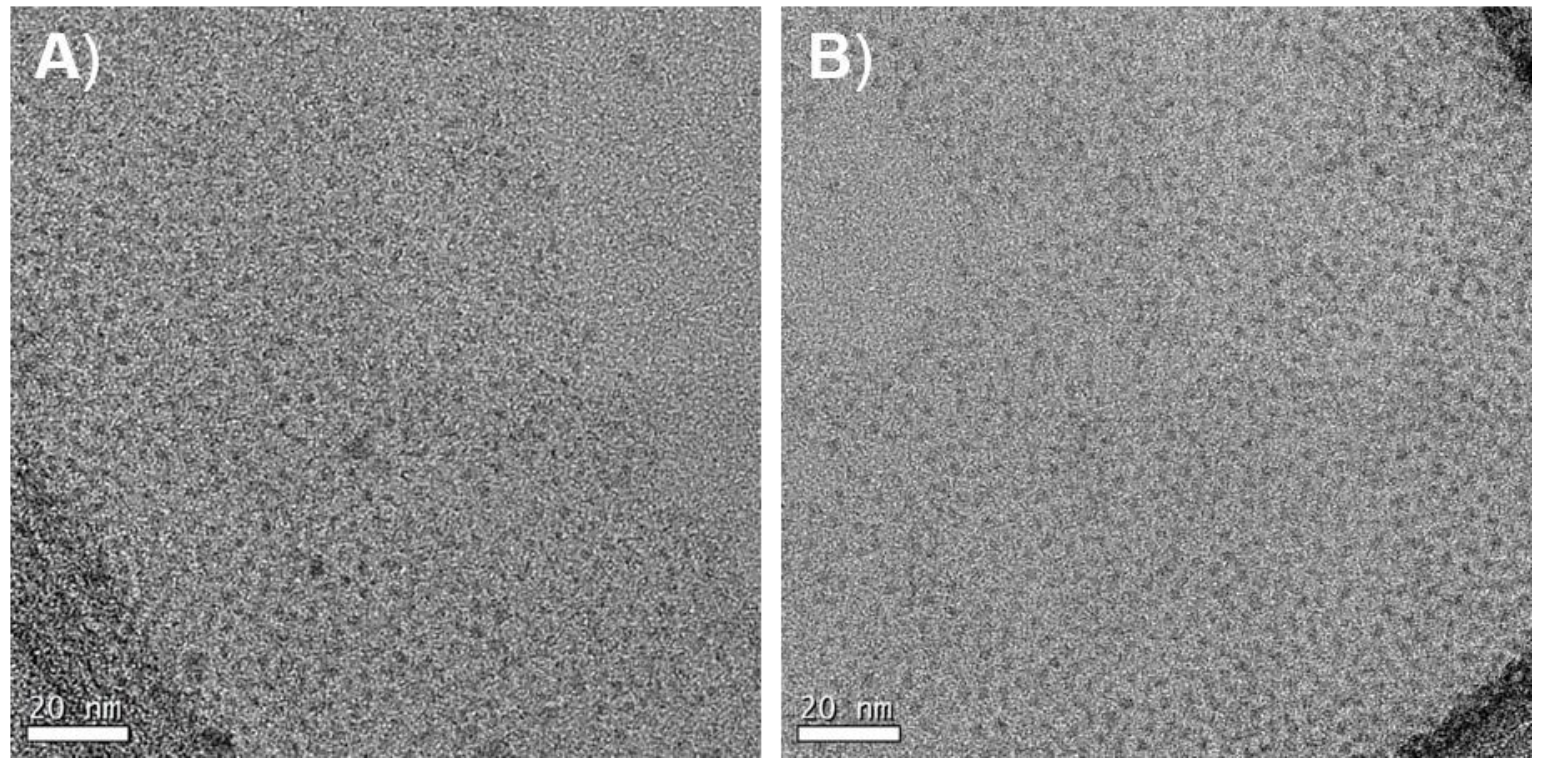

Figure S4. TEM images of $\mathrm{Cu}^{+}: \mathrm{InP}$ NCs synthesized from $\operatorname{InBr}_{3}(\mathbf{A})$ and $\operatorname{InI}_{3}(\mathbf{B})$. 

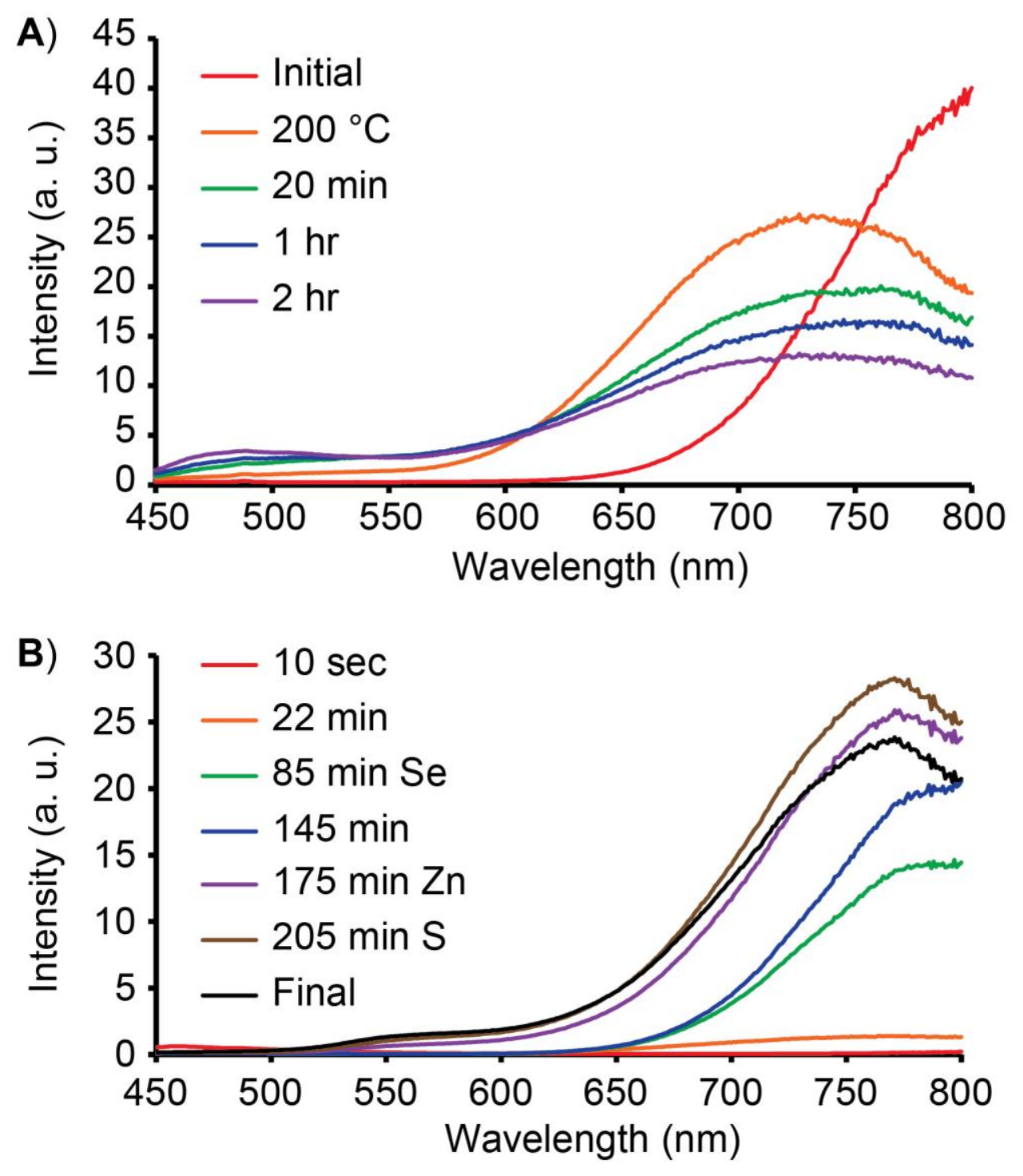

Figure S5. Photoluminescence spectra of copper doped samples during standard Lewis acid treatment with zinc stearate $(\mathbf{A})$ and using a modified $\mathrm{ZnSeS}$ shelling procedure $(\mathbf{B})$. Both spectra show the emergence of excitonic luminescence below $600 \mathrm{~nm}$. 
Table S1. ICP-OES elemental ratios of nanocrystals formed under various synthetic conditions.

\begin{tabular}{|c|c|c|c|c|c|}
\hline Sample & Indium & Phosphorous & Copper & Zinc (Cadmium) & Selenium \\
\hline $\mathrm{InCl}_{3}$ 10:1 In:Cu & 1.2 & 1 & 0.07 & -- & $\begin{array}{l}-- \\
-1\end{array}$ \\
\hline $\mathrm{InCl}_{3}$ 20:1 In:Cu & 1.3 & 1 & 0.08 & -- & -- \\
\hline $\mathrm{InCl}_{3}$ 40:1 In: $\mathrm{Cu}$ & 1.2 & 1 & 0.04 & -- & -- \\
\hline $\mathrm{InBr}_{3}$ & 1 & 1 & 0.1 & -- & -- \\
\hline $\mathbf{I n I}_{3}$ & 1 & 1 & 0.13 & -- & -- \\
\hline Zinc treated & 1 & 1 & 0.07 & 0.8 & -- \\
\hline Cadmium treated & 1 & 1 & 0.05 & $(0.7)$ & -- \\
\hline Thin shelled & 1.3 & 1 & 0.1 & 0.2 & 0.8 \\
\hline $\mathrm{CuBr}_{2}$ & 1 & 1 & 0.25 & -- & -- \\
\hline
\end{tabular}
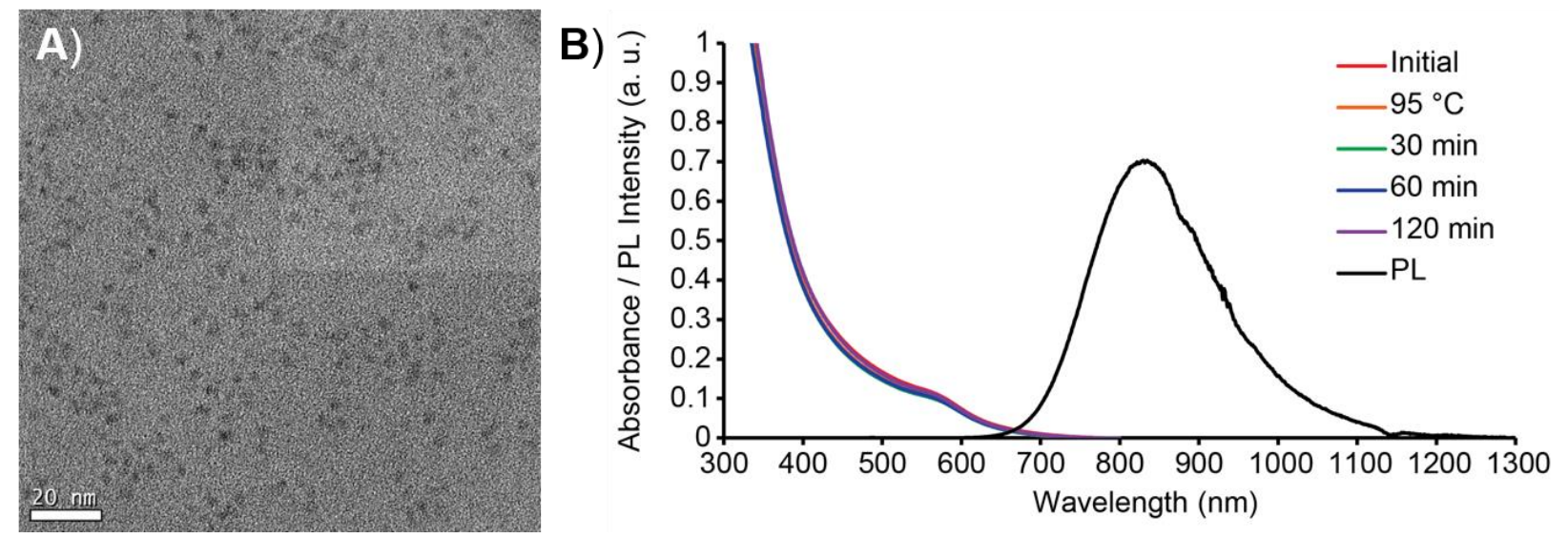

Figure S6. TEM of zinc carboxylate treated Cu:InP NCs (A) and UV-Vis and final PL spectrum showing the continued presence of copper in the nanocrystal (B). 

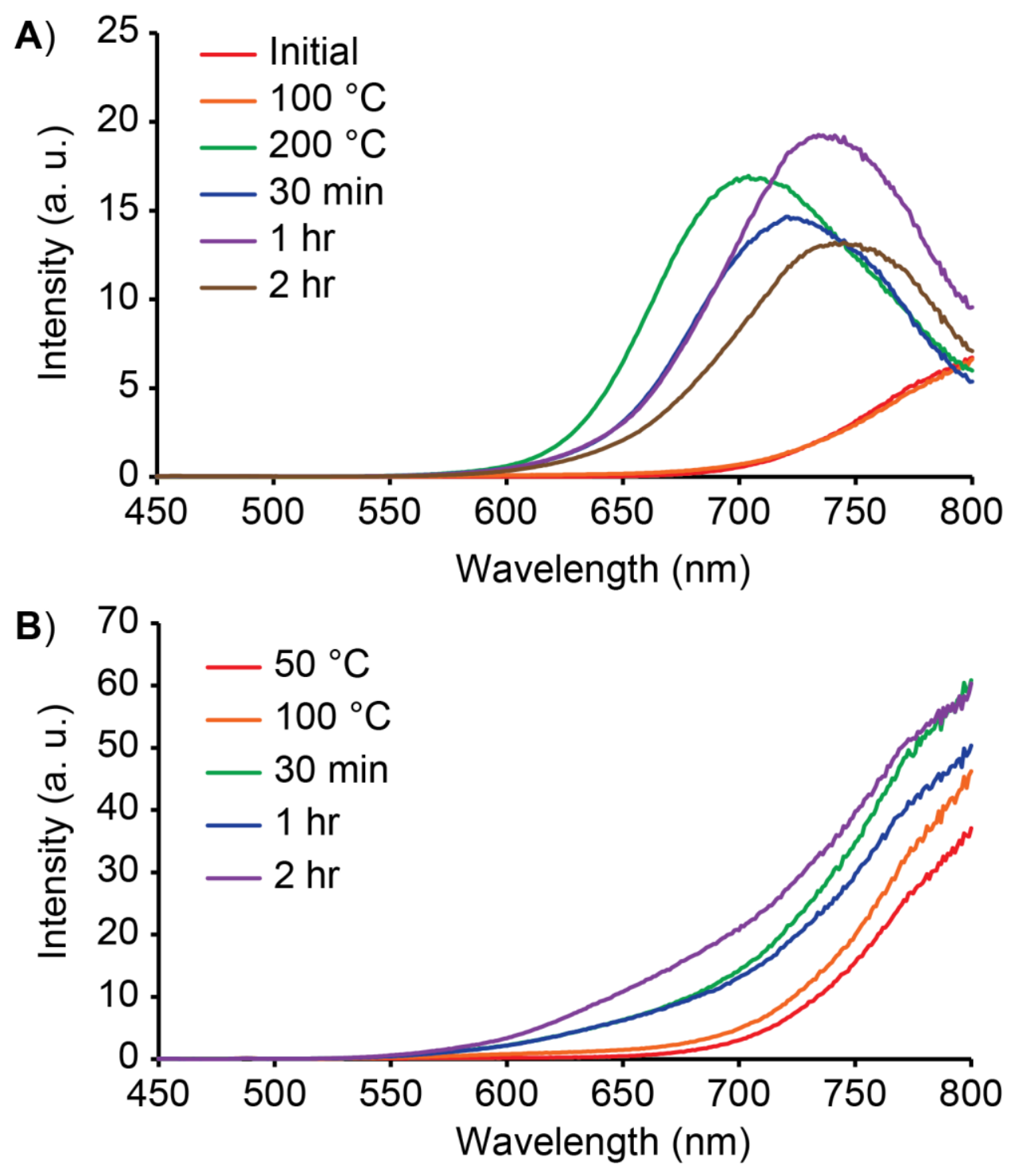

Figure S7. Photoluminescence spectra of $\mathrm{Cu}^{+}: \mathrm{InP}$ NCs after cadmium oleate treatment at $200{ }^{\circ} \mathrm{C}(\mathrm{A})$ or $100{ }^{\circ} \mathrm{C}(\mathbf{B})$. We attribute the increase in emission near 650-700 $\mathrm{nm}$ to the red shifted excitonic emission characteristic of Cd-treated InP as previously demonstrated in J. Phy. Chem. Lett. 2016, 7, 1315-1320. ICP analysis of the final sample gives a ratio of In:P:Cu:Cd of 1:1:0.05:0.7. This confirms a decrease in $\mathrm{Cu}$ content over the course of the reaction relative to the starting $\mathrm{Cu}^{+}: \mathrm{InP}$ NCs which have a In:Cu ratio of 1:0.08. 

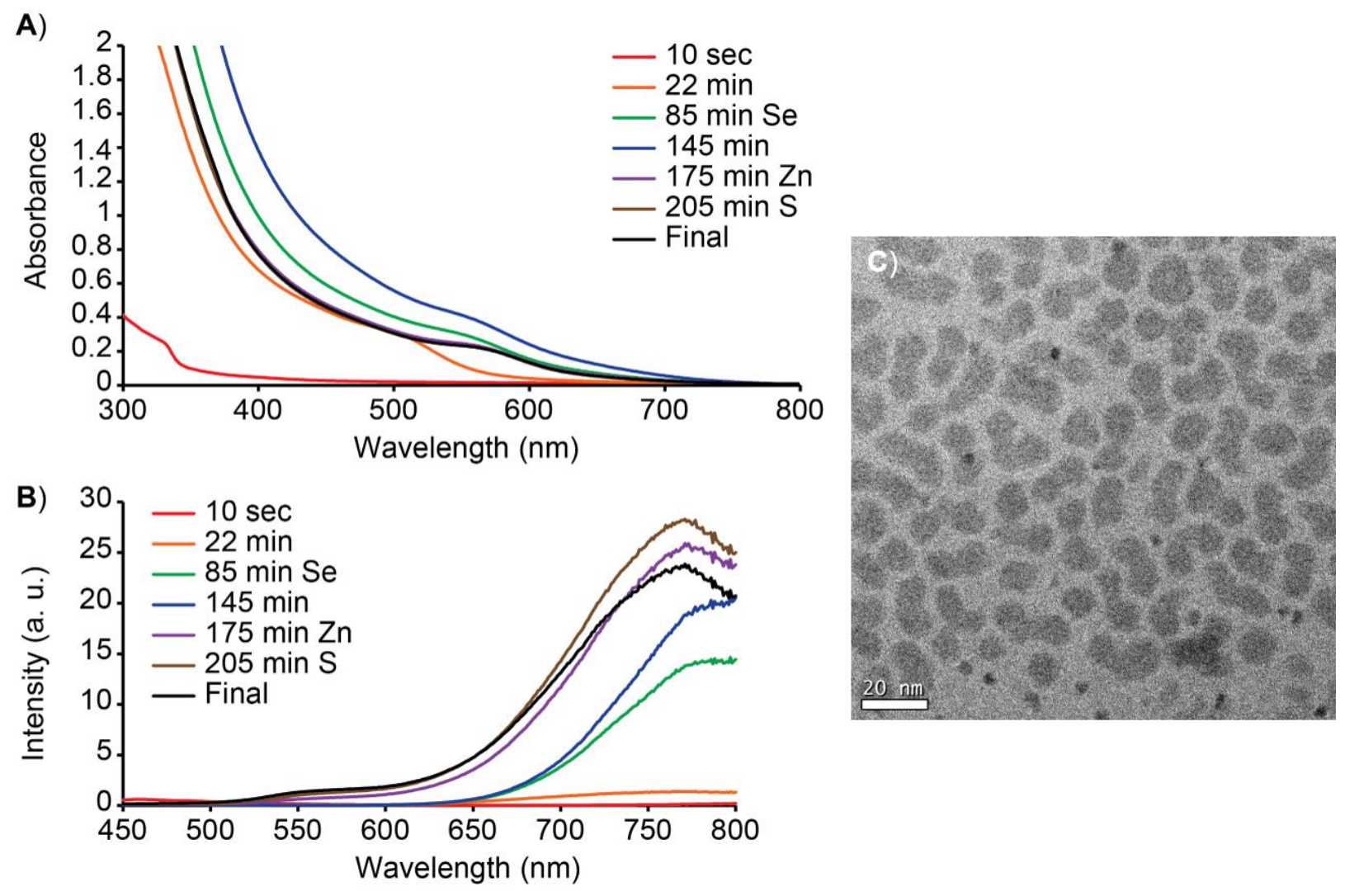

Figure S8. Absorption spectra of $\mathrm{Cu}^{+}$:InP NCs thickly shelled in-situ with ZnSeS. The UV-Vis (A) shows the development of sub-band gap absorbance associated with the so-called "copper foot" and retention of the InP excitonic absorption while the PL spectra (B) show both the distinct copper luminescence and the presence of excitonic luminescence around $565 \mathrm{~nm}$. (C) Shows a representative TEM image of the sample confirming the deposition of a thick shell on the InP NCs. From the measured average diameter of about $8.5 \mathrm{~nm}$, we estimate a shell thickness of about 8 monolayers $(\sim 0.33 \mathrm{~nm} / \mathrm{ML} \mathrm{ZnSe})$. 

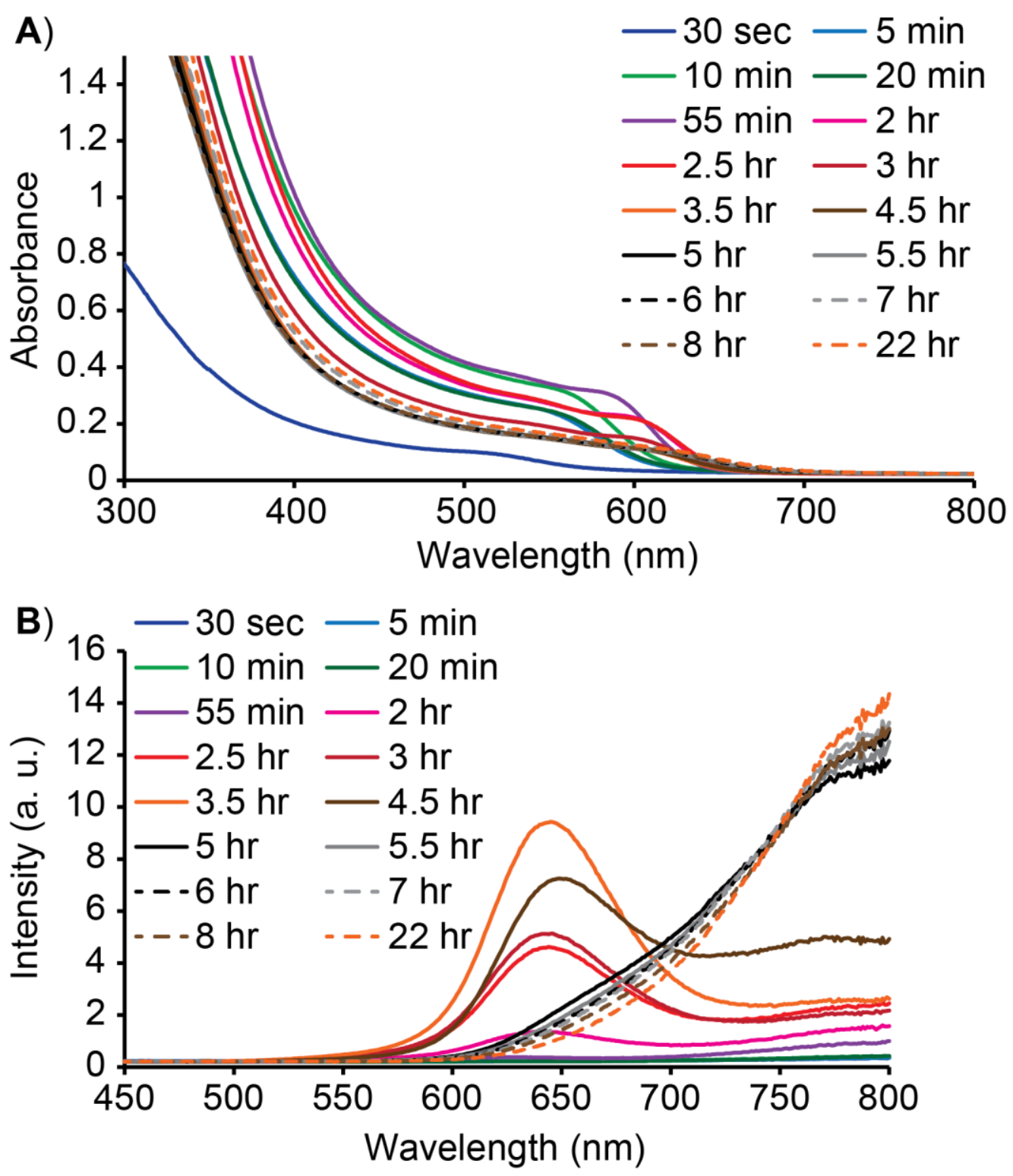

Figure S9. Absorption and PL spectra collected during post-synthetic treatment of thickly shelled $\mathrm{InP} / \mathrm{ZnSeS} \mathrm{NCs}$ with a copper chloride and oleylamine solution at $210{ }^{\circ} \mathrm{C}$ for a period of $22 \mathrm{~h}$. The UVVis absorption spectra (A) show the development of sub-band gap absorbance associated with the socalled "copper foot" while the PL spectra (B) show the diminishment of the excitonic luminescence and the emergence of the distinct copper luminescence. 


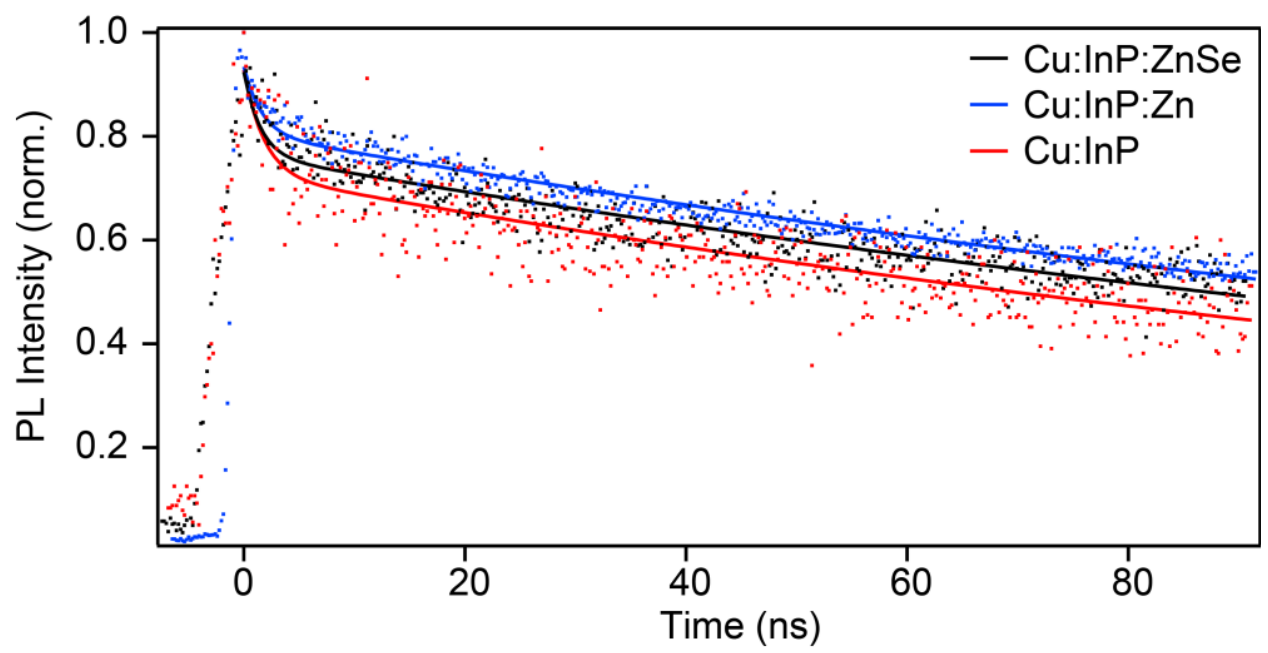

Figure S10. Time resolved copper luminescence decay dynamics (dots are data and solid lines are fits), illustrating a nanosecond trapping component that the $\mu$ s timescale measurements do not capture. The $<10$ ns process associated with carrier trapping persists upon surface treatment. All data were collected at room temperature using NCs suspended in dry toluene. PL decay curves were obtained by integrating between 880 and $780 \mathrm{~nm}$.

Table S2. Fitting parameters for time resolved photoluminescence decay dynamics ( $1 \mu$ s fitting window Figure 3 of main text).

\begin{tabular}{|c|c|c|c|c|c|}
\hline & $\mathbf{A 1}$ & $\tau 1(\mu \mathrm{s})$ & $\mathbf{A} 2$ & $\tau 2(\mu \mathrm{s})$ & Weighted $\tau(\mu \mathrm{s})$ \\
\hline $\mathrm{Cu}^{+}: \mathrm{InP}: \mathrm{ZnSe}$ & 0.098 & 0.036 & 0.913 & 0.415 & 0.383 \\
\hline $\mathrm{Cu}^{+}: \mathbf{I n P}: \mathrm{Zn}$ & 0.315 & 0.078 & 0.632 & 0.430 & 0.313 \\
\hline $\mathrm{Cu}^{+}: \mathrm{InP}$ & 0.378 & 0.092 & 0.588 & 0.405 & 0.272 \\
\hline
\end{tabular}

Table S3. Fitting parameters for TRPL decay dynamics (100ns fitting window - Figure S9). The longer time component values are not physical given the small magnitude of the overall decay observed in this window.

\begin{tabular}{llllll} 
& $\mathbf{A 1}$ & $\boldsymbol{\tau 1}(\mathbf{n s})$ & $\mathbf{A 2}$ & $\boldsymbol{\tau} \mathbf{2}(\mathbf{n s})$ & Weighted $\boldsymbol{\tau}(\mathbf{n s})$ \\
\hline $\mathbf{C u}{ }^{+}:$InP:ZnSe & 0.15968 & 1.4931 & 0.32784 & 46.629 & 70.1 \\
$\mathbf{C u}^{+}:$InP:Zn & 0.12851 & 1.5864 & 0.39505 & 72.579 & 54.9 \\
$\mathbf{C u}^{+}:$InP & 0.20106 & 1.8231 & 0.36808 & 61.17 & 39.9
\end{tabular}

All traces were fit to the biexponential equation S1:

$$
A=A_{1} * \exp \left[-\frac{t}{\tau_{1}}\right]+A_{2} * \exp \left[-\frac{t}{\tau_{2}}\right]
$$

Weighted lifetimes were calculated using the equation S2:

$$
\tau_{\text {weighted }}=\frac{\left[\left(A_{1} \tau_{1}\right)+\left(A_{2} \tau_{2}\right)\right]}{\left(A_{1}+A_{2}\right)}
$$




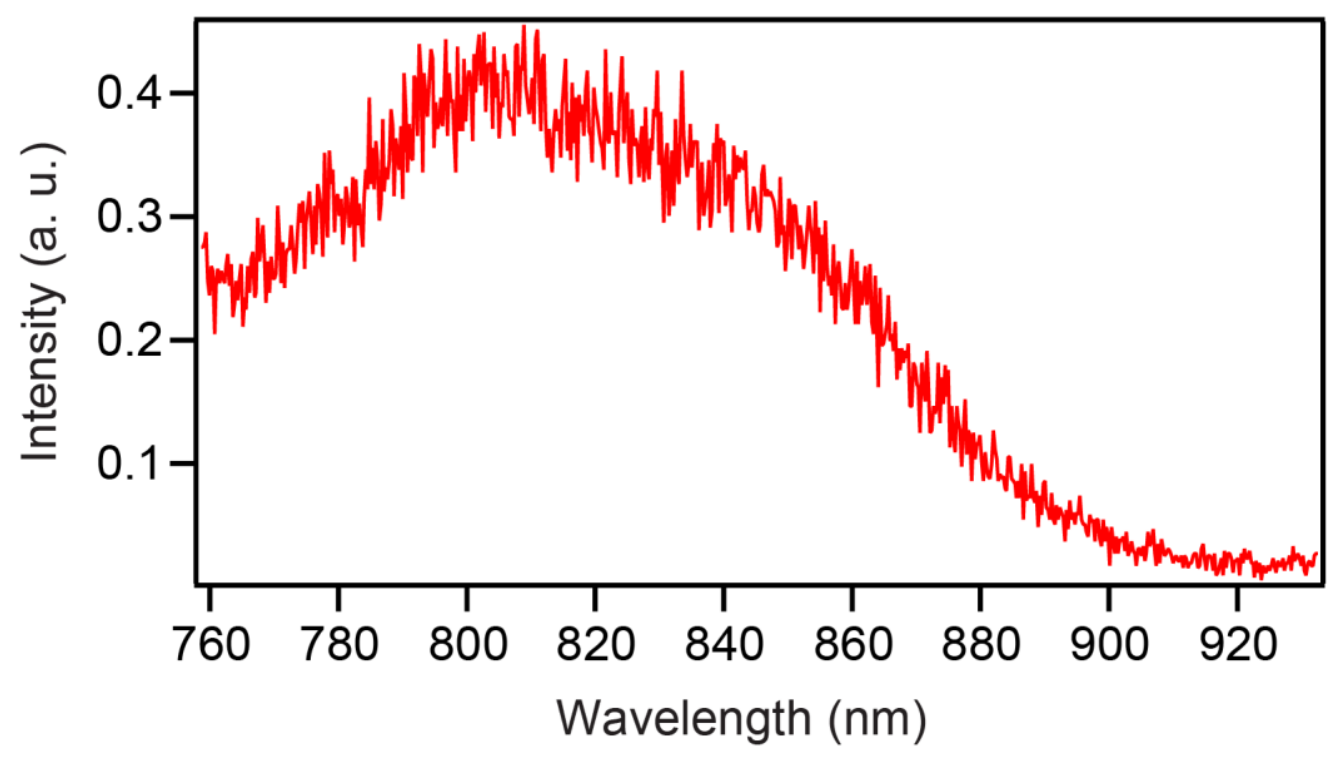

Figure S11. PL spectrum of $\mathrm{Cu}^{+}: \mathrm{InP}$ NCs, obtained by integrating streak camera data over the first $1 \mu$ s.

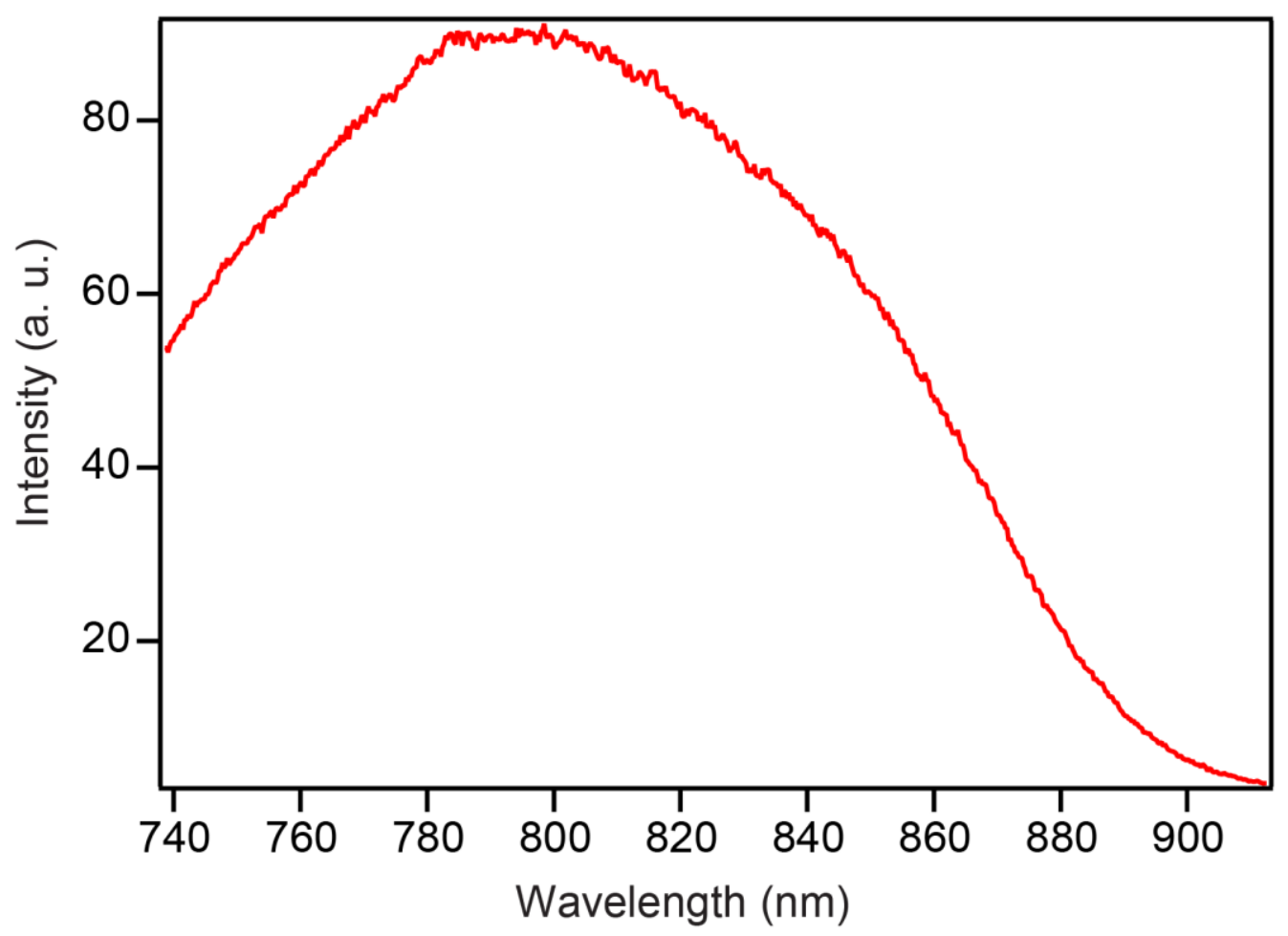

Figure S12. PL spectrum of $\mathrm{Cu}^{+}: \mathrm{InP} / \mathrm{Zn}$ NCs obtained by integrating streak camera data over the first 1 $\mu \mathrm{s}$. 


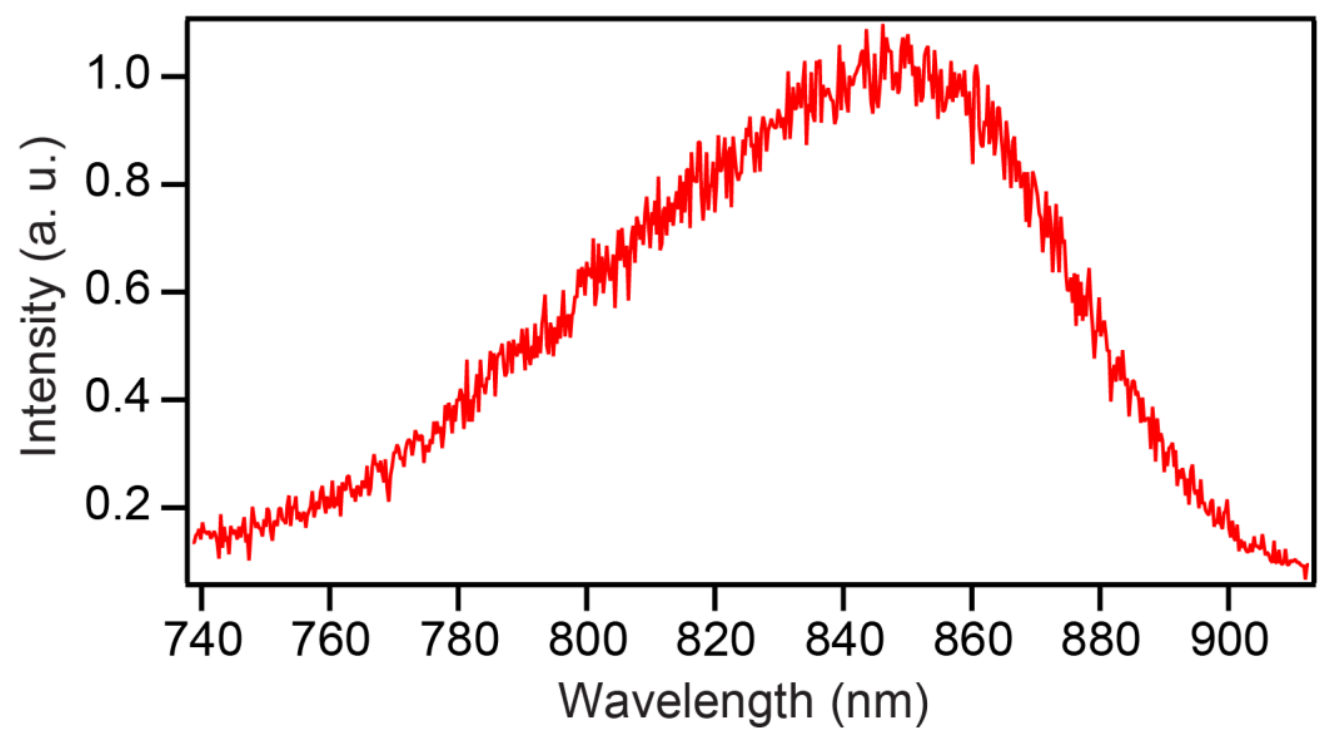

Figure S13. PL spectrum of $\mathrm{Cu}^{+}: \mathrm{InP} / \mathrm{ZnSe}$ NCs, obtained by integrating streak camera data over the first $1 \mu \mathrm{s}$.

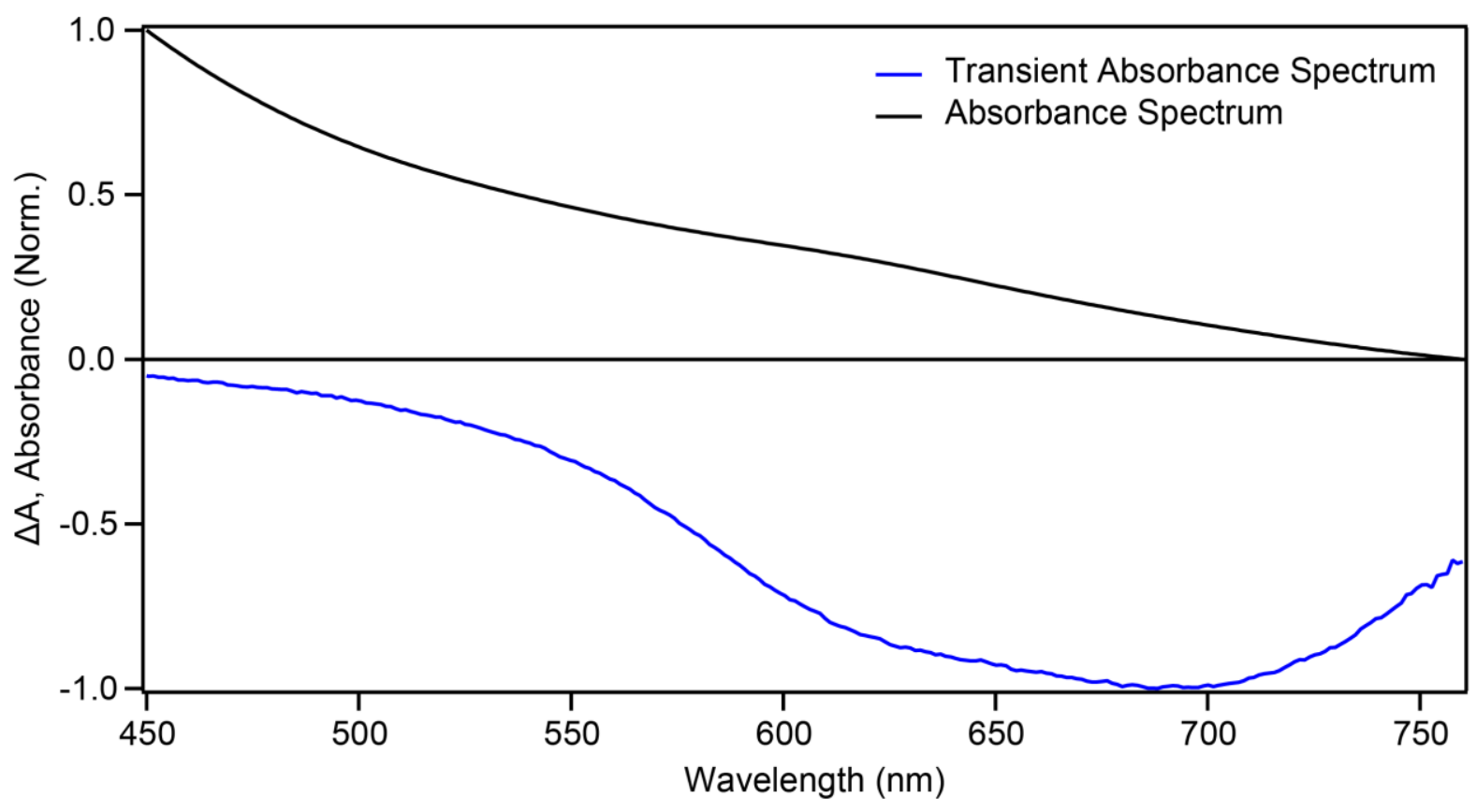

Figure S14. Comparison of Transient Absorption spectrum with absorbance spectrum of $\mathrm{Cu}^{+}: \mathrm{InP} / \mathrm{ZnSe}$ NCs. TA data was normalized to excitonic bleach, while absorbance data was normalized to $450 \mathrm{~nm}$. 


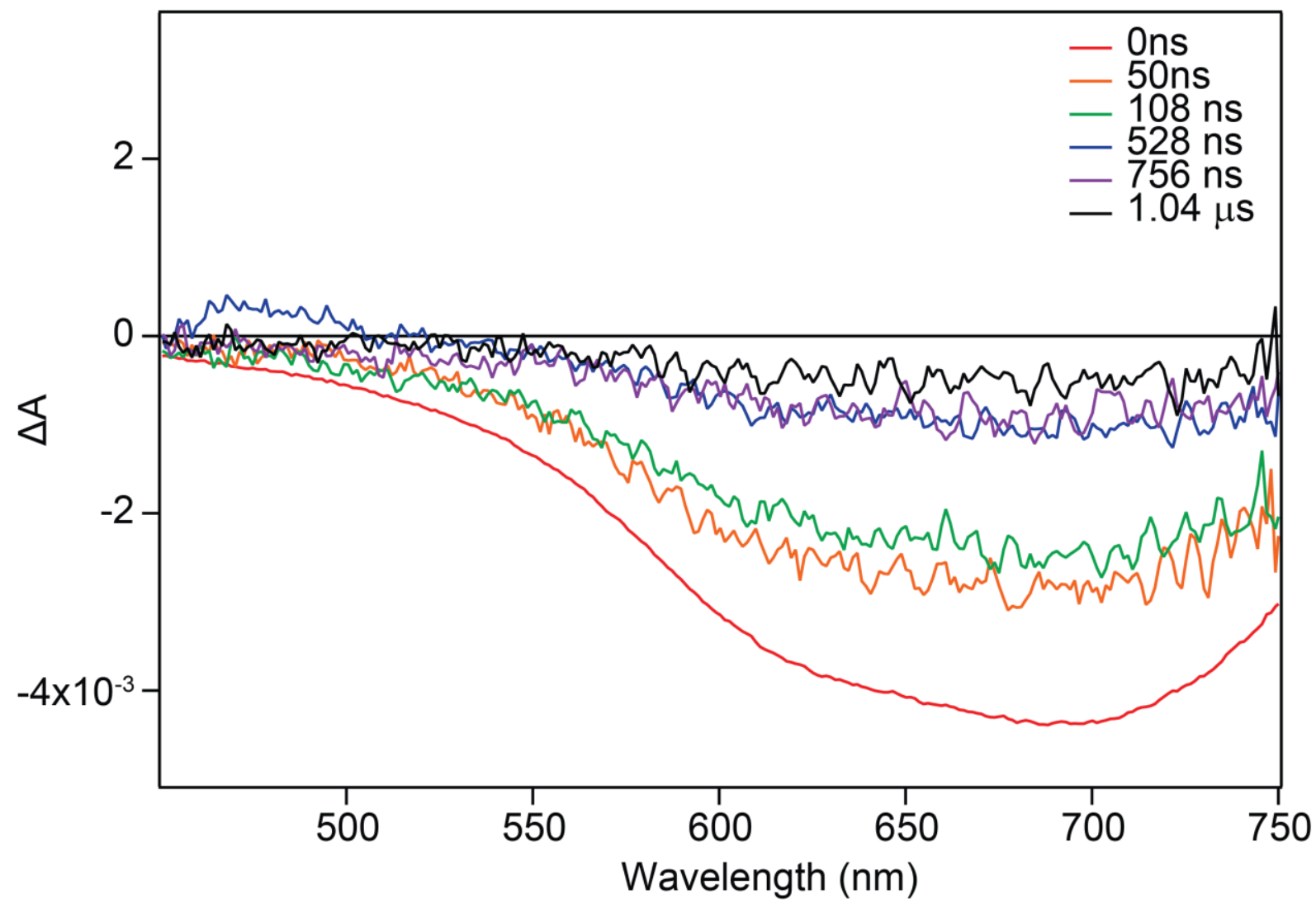

Figure S15. Temporal evolution of $\mathrm{Cu}^{+}: \mathrm{InP} / \mathrm{ZnSe}$ TA spectrum. All data were collected at room temperature on NCs suspended in toluene. 


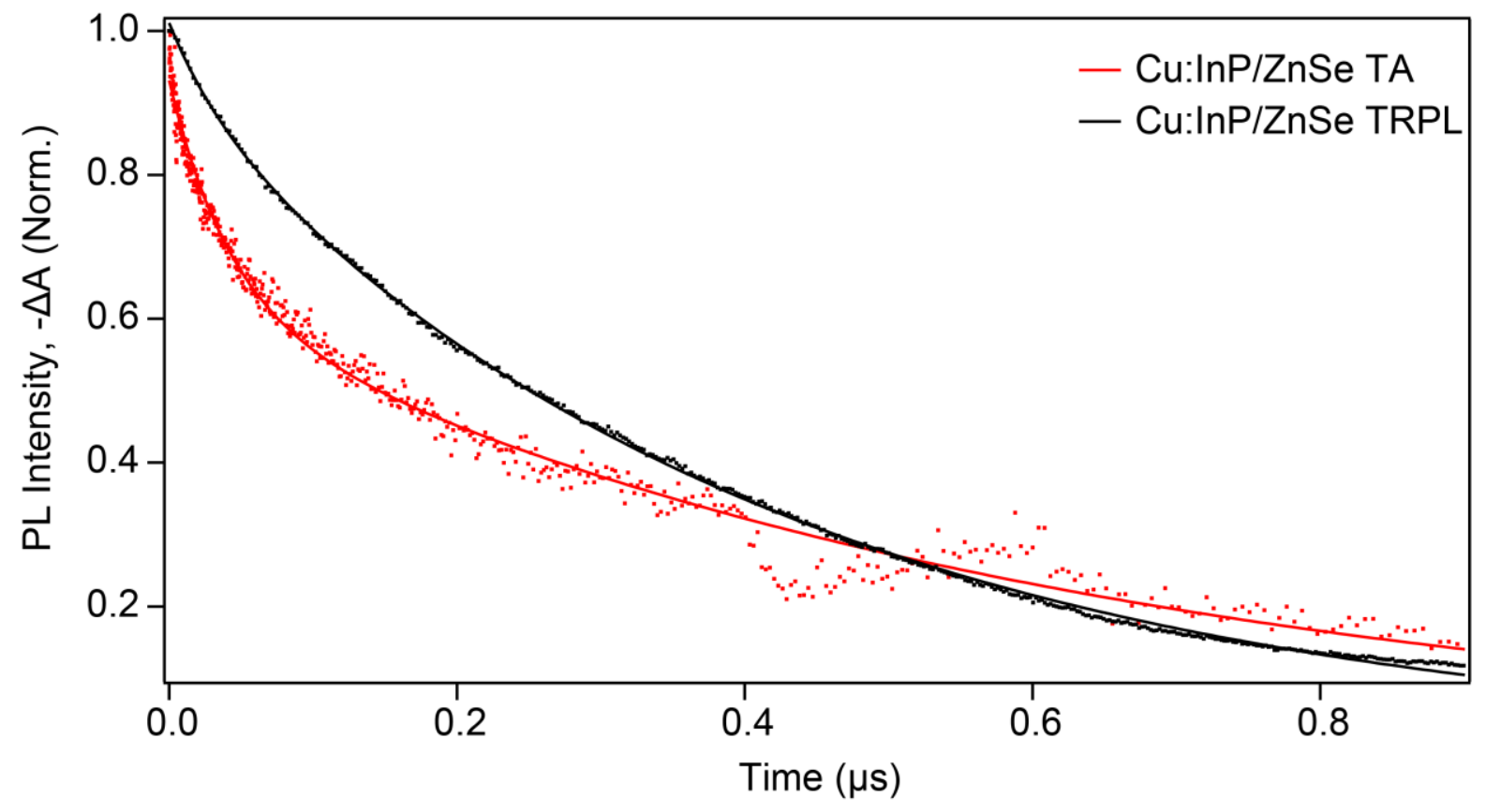

Figure S16. Normalized room temperature excitonic TA bleach recovery (red) in comparison to copper TRPL decay (black). All data were collected at room temperature on NCs suspended in toluene (dots are data and solid lines are fits).

Table S4. Fitting parameters for TA bleach over $1 \mu \mathrm{s}$. The bleach recovery was fit to the biexponential equation S1; weighted lifetime was calculated using the equation described in equation $\mathrm{S} 2$.

$\begin{array}{llllll} & \mathbf{A 1} & \boldsymbol{\tau} \mathbf{1}(\mu \mathrm{s}) & \mathbf{A 2} & \boldsymbol{\tau} \mathbf{2}(\mu \mathrm{s}) & \text { Weighted } \boldsymbol{\tau}(\mu \mathrm{s}) \\ \mathbf{C u}^{+}: \text {InP:ZnSe } & 0.306 & 0.041 & 0.626 & 0.603 & 0.419\end{array}$

